\title{
Numerical analysis of the thermal behaviour of a photovoltaic panel coupled with phase change material
}

\author{
G. Santiago-Rosas ${ }^{1,2}$, M. Flores-Domínguez ${ }^{2}$, J. P. Nadeau ${ }^{2}$ \\ ${ }^{1}$ Center for Research and Advanced Studies, National Polytechnic Institute, Mexico City, Mexico \\ ${ }^{2}$ Trefle Laboratory, Arts and Trade Paris-Tech (ENSAM), Talance, France
}

\section{Email address:}

mfdguez@gmail.com (M. Flores-Domínguez)

\section{To cite this article:}

G. Santiago-Rosas, M. Flores-Domínguez, J. P. Nadeau. Numerical Analysis of the Thermal Behaviour of a Photovoltaic Panel Coupled with Phase Change Material. Science Journal of Energy Engineering. Vol. 2, No. 5, 2014, pp. 58-64. doi: 10.11648/j.sjee.20140205.12

\begin{abstract}
Elevated operating temperatures reduce the solar to electrical conversion efficiency of photovoltaic devices. In this paper, a numerical study is conducted to understand the thermal behavior of a photovoltaic panel with phase change materials. The analysis of the PV/PCM system was realized in three phases: temperature rise, start of melting of PCM and molten PCM so, three mathematical models for the resolution of thermal model of PV/PCM system during the solar insolation were used. First, an analytical model was used to study the behavior of PCM in state solid, after, an explicit and an implicit model were used to study globally the transfers. Different simplifications are introduced in the heat balances to realize a simple numerical approach. The Matlab software was used to solve different proposed models in this analysis. The results obtained are considered as a first approach to the study of a PV/PCM system.
\end{abstract}

Keywords: Photovoltaic Panel, Phase Change Material, Numerical Analysis

\section{Introduction}

The production of clean energy has allowed the development of the photovoltaic industry. The photovoltaic devices operate at elevated temperature and it reduces their efficiency. Similarly, the solar panels are simultaneously a heat source that penalizes the production of electric power due to the fact that, during operation of the photovoltaic cell, only about $15 \%$ of the solar radiation that incident on them is transformed into electricity and the remainder is transformed into heat. So that, the performance of the photovoltaic modules is closely related to its temperature [1].

The most important research works in solar cells focus on its performance, as production decreases as its temperature increases. The loss coefficient is estimated at approximately $0.45 \%$ per degree Celsius of increase, with reference to $25^{\circ} \mathrm{C}$ [2].

In order to use solar cells at low operating temperature, researchers have focused on cooling and recovery of the dissipated heat. However, despite all efforts realized for optimizing the performance of solar panels, the actual temperature reaching is still not enough, so that the relentless pursuit of solutions for cooling continues nowadays.

New materials and systems have been studied in cooling of panels, such is the case of the phase change materials (PCMs).
The PCMs absorb a large amount of energy as latent heat at a constant phase transition temperature and are thus used for passive heat storage. The thermal energy transfer occurs when the material changes from solid to liquid, or liquid to solid phase. The PCMs are of great interest for their use in various applications as the thermal energy storage and the thermal control systems. The energy storage capacity of the PCMs is dependent on its properties, methods of heat transfer and the system configuration [3].

The PCM cooling is a natural method used since antiquity in the cooling of buildings with thermal energy storage [4]. When there is a temperature gradient within a system exists a transfer of energy that allows release or transfer of energy in the zone of state change. The advantage in this fact lies in that is possible to store large amounts of thermal energy in relatively small masses and with small variations of temperature.

To incorporate the PCM into the photovoltaic (PV) panel to form a PV/PCM system can allow that the efficiency of the conversion of solar power of the panel to be closer at characterization conditions of the standard cell. In the study conducted by Huang [5] was validated numerically that use of PCM in photovoltaic panels moderates the increase of temperature of thereof. The model gives a detailed explanation of the thermal performance of the phase transition 
from the solid to the liquid state of the material.

In this work, a numeric analysis to understand the thermal behavior of a photovoltaic panel with material change of phase is proposed. The thermal analysis of PV/PCM system to know the behavior of the temperature of the panel was realized. The system analysis was realized in three phases: 1) temperature rise, 2) start of melting of PCM and, 3) molten PCM; so that, three mathematical models for the resolution of thermal model of PV/PCM system were used. First, an analytical model was used to study the behavior of PCM solid state, after, an explicit and an implicit model were used to study the transfers jointly. The Matlab software was used to solve different proposed models in this analysis.

\section{Modeling of System}

A schematic of the system configuration discussed in this paper is shown in Fig. 1. The system is comprised of a photovoltaic panel and of a material change of phase. Air circulates freely over the PV panel, the PCM is in direct contact with the PV panel and, the back wall of the container of PCM is considered adiabatic. The solar insolation received by the PV panel during the day is a sinusoidal function, with a maximum value of $750 \mathrm{Wm}^{-2}$ [6].

The physical phenomena taken into account for this analysis were:

- Sun/PV interaction: Solar radiation.

- Air/PV interaction: Natural convection.

- PV: Source of heat due to the operation of the photovoltaic cell (Conduction).

- Liquid PCM: Conduction (natural convection negligible).

- PV/Solid PCM Interface: Start of melting.

- Liquid PCM/Solid PCM Interface: phase change.

- PCM/Air Interaction: Heat transfer does not exist, adiabatic wall.

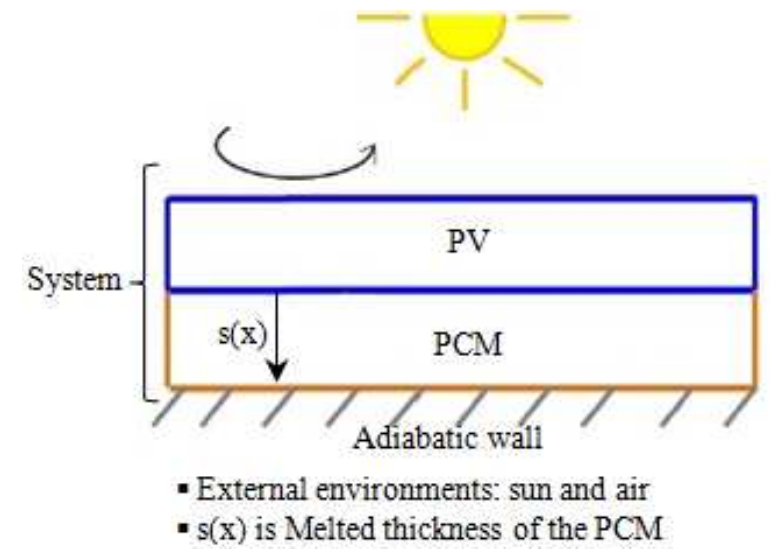

Figure 1. Schematic representation of PV/PCM system

The system analysis was carried out in three phases: Phase 1: Rise of temperature.

At the beginning, the PV panel and the solid PCM are considered that are at ambient temperature. The PV panel temperature increases over time due to the absorption of the solar energy it receives directly on its front surface. This energy is converted into heat and is transferred by conduction to the solid PCM whose temperature also increases. In this phase, the system was analyzed through an analytical model, where the hypothesis of a uniform temperature in the solid PCM was made. Furthermore, the hypothesis of $T_{P C M s}=T_{P V}$ was raised.

\section{Phase 2: Start of melting}

Once the solid PCM reached the melting temperature, its temperature depends on the molten fraction of the same one. This temperature remains almost constant during the period required for that phase-change process is performed. Thus, to analyze the behavior of the temperatures in this phase, the hypothesis of a constant temperature in the PV/PCM interface was posed, and thus, an explicit method was used. For a more accurate approximation, an implicit method that allows to follow the actual behavior of the temperature of the material was used. To simplify calculations, the hypothesis of uniformity of the temperature of the liquid PCM was made.

Phase 3: Melted PCM

Finally, when there is no more PCM in solid state, its temperature begins to increase, and the temperature of the PV depends on the conductive transfer with the PCM in liquid state.

For the resolution of thermal model of PV/PCM system different hypothesis were raised. The models are valid if the following hypothesis are true.

1 The PCM is in direct contact with the PV.

2 The temperature at the liquid/solid interface is constant in the explicit model and is uniform in the implicit model.

3 The latent heat is constant.

4 The container is adiabatic.

5 The ambient temperature is $21 \pm 1{ }^{\circ} \mathrm{C}$.

6 There is no convection in the liquid PCM (the liquid $\mathrm{PCM}$ is viscous $\rightarrow$ conductive transfer).

\section{Analysis of the System}

\subsection{Analytical Model}

In this section, the development of the temperature of the PCM solid state is analyzed. Considering that the temperature is almost uniform in the material, the only variables were the time and the temperature of the PCM and PV. For the analysis of the system, the resistance to heat transfer of the specimen was characterized, that is, the thermal resistance of the surface and the internal conduction resistance of the specimen was compared [7]. The dimensionless Biot number (Bi), which compares the convective flow (boundary condition) with the conductive flux that matter can emit or absorb, is used. If $\mathrm{Bi}<0.1$, then the hypothesis of temperature uniformity in the specimen is verified.

For the proposed system, the value of $\mathrm{Bi}=03$ is used, so that it was necessary to take into account the temperature gradient existing in the first hours of solar insolation (when the PCM is even solid). 
The analysis to estimate, roughly, the value of the temperature gradient was carried out in this section. An analytical model that used the method of the Laplace transform was used and, to represent the temperature change with respect to time was possible.

Through the Stehfest method combined with the thermal quadrupole method, the simple heat equation is a linear partial differential equation of parabolic type, where the particular solutions can be overlapped to obtain the general solution of the system [8].

If the case of unidirectional heat transfer through a wall of thickness $e$ is considered, a thermal quadrupole method for the analysis of this particular case can be used, as shown in Fig. 2.

In general, it is observed that there is a linear relationship

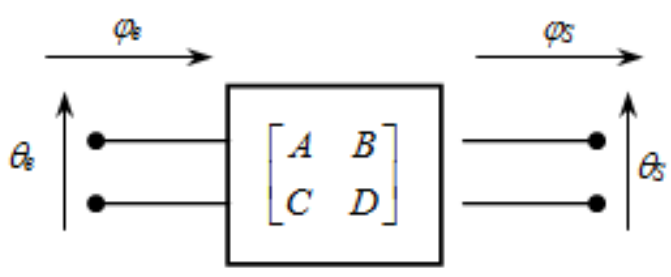

Figure 2. Quadrupole of a passive wall

between the input values and the output values, given by:

$$
\left[\begin{array}{l}
\theta_{e} \\
\varphi_{e}
\end{array}\right]=\left[\begin{array}{ll}
A & B \\
C & D
\end{array}\right]\left[\begin{array}{l}
\theta_{e} \\
\varphi_{e}
\end{array}\right]
$$

where $\theta_{e}$ and $\theta$ s are the Laplace transform of temperature T at $x=0$ and $x=e$ respectively; $\varphi_{e}$ and $\varphi_{s}$ are the Laplace transform of flow $Q$ en $x=0$ and $x=e$ respectively; and the matrix is the reverse transfer matrix of the quadrupole associated to wall. For the particular case of study, is given by the following:

Balance of solid PCM

The configuration of the system is shown in Fig. 3. The flow $\phi(t)$ directly affects the PV, which is designated with the number 1 . The panel/material interface is denoted by the number 2 and, the adiabatic wall is the number 3 .

From the linear relation shown in (1), the terms of the matrix are defined as:

$$
\begin{gathered}
A=D=\cosh (\alpha e) \quad, \quad B=\frac{1}{\lambda A} \frac{\sinh (\alpha e)}{a} \\
C=\lambda A \sinh (\alpha e)
\end{gathered}
$$

with

$$
\alpha=\sqrt{\frac{p}{a}} \text { and } p=\frac{j \ln (2)}{t}
$$

where $\lambda$ is heat conductivity, $p$ is Laplace variable, and $a$ is thermal diffusivity.

Thus, (2a) and (2b) are, respectively, for the wall 1 and 2, these are:

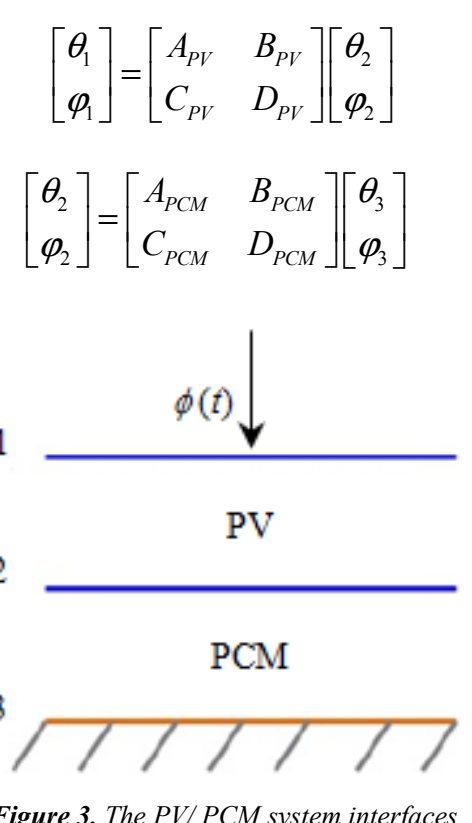

Figure 3. The PV/PCM system interfaces

where $\varphi_{3}=0$ since the system is adiabatic on the side of the PCM, the equation can be rewritten as follows:

$$
\left[\begin{array}{l}
\theta_{1} \\
\varphi_{1}
\end{array}\right]=\left[\begin{array}{ll}
A_{P V} & B_{P V} \\
C_{P V} & D_{P V}
\end{array}\right]\left[\begin{array}{ll}
A_{P C M} & B_{P C M} \\
C_{P C M} & D_{P C M}
\end{array}\right]\left[\begin{array}{l}
\theta_{3} \\
0
\end{array}\right]
$$

Solving for $\theta_{3}$, yield:

$$
\theta_{3}=\frac{\varphi_{1}}{\left(C_{P V} A_{P C M}+A_{P V} C_{P C M}\right)}
$$

and because the flow that is received by the panel is of sinusoidal form, $\theta_{3}$ is:

$$
\theta_{3}=\frac{\varphi_{1}}{\left(C_{P V} A_{P C M}+A_{P V} C_{P C M}\right)} \frac{\omega}{p^{2}+\omega^{2}}
$$

\section{Balance of $P V$}

To calculate the temperature of the PV/PCM interface, the flow in the wall 2 was used, which is represented by ( $2 b)$, that is:

$$
\theta_{2}=A_{P C M} \theta_{3}
$$

\subsection{Explicit Model}

In this section, the behavior of the temperature of the PV panel and of the PCM with respect to the time was analyzed, so the explicit model was divided into three stages:

\subsubsection{When $T_{P C M}=T_{P V}$}

Initially, the temperature of the PV panel $\left(T_{P V}\right)$ is equal to the ambient temperature $\left(T_{a m b}\right)$ and, the temperature of the solid PCM $\left(T_{P C M}\right)$ is equal to the temperature of the panel.

Energy balance in the $P V$

(It was considered that there is no variation in the temperature of the solid PCM). When the temperature of the $\mathrm{PV}$ panel is less than the melting temperature $\left(T_{P V}<T_{M}\right)$, the 
difference between the source of energy of the system and the loss in the air/PV interface is the variation of the thermal mass, this is:

$$
\begin{gathered}
(1-\eta) A I-A h\left(T_{P V}-T_{a m b}\right)= \\
\left(M_{P V} C p_{P V}+M_{P C M s} C p_{P C M s}\right) \frac{\mathrm{d} T_{P V}}{\mathrm{~d} t}
\end{gathered}
$$

where $A$ is the surface area, $I$ is the incident energy absorbed by the PV/PCM system, $h$ is the coefficient of natural convection, $M$ is the mass, and $C p$ is the specific heat capacity.

Balance of heat energy in the PCM

$$
\begin{gathered}
T_{P C M}=T_{P V} \\
s(x)=0
\end{gathered}
$$

\subsubsection{When $T_{P V}=T_{M}$}

In this stage, the PV panel reaches the melting temperature of the PCM. The PCM begins to melt.

Energy balance in the PV panel

The difference between the source of energy, the loss in the air/PV interface and the transfer at the PV/PCM interface is the variation of the thermal mass, that is:

$$
\begin{gathered}
(1-\eta) A I-A h\left(T_{P V}-T_{a m b}\right)-A \frac{\lambda_{L}}{s(x)}\left(T_{P V}-T_{M}\right)= \\
\left(M_{P V} C p_{P V}\right) \frac{\mathrm{d} T_{P V}}{\mathrm{~d} t}
\end{gathered}
$$

\section{Balance of heat energy in the PCM}

(It was considered that there is no sensitive heat in the liquid PCM). When $T_{P C M}=T_{M}$, the difference between the source of energy and the loss in the loss in the air/PV interface is transfer at the $\mathrm{PV} /$ liquid $\mathrm{PCM}$ interface, this is:

$$
(1-\eta) A I-A h\left(T_{P V}-T_{a m b}\right)=\frac{s(x)}{\mathrm{d} t} A \rho_{L} \Delta h_{M}
$$

where $\rho$ is the reference density.

\subsubsection{When $T_{P C M}>T_{F}$}

Finally, when the volume of the solid PCM has melted its temperature begins to rise and, as result, the temperature of the panel also increases due to conductive transfer that exist between the liquid PCM and the PV panel, is given as:

$$
T_{P C M_{L}}=\left(T_{P V}+T_{M}\right) / 2
$$

\subsection{Implicit Model}

To increase the accuracy in the analysis, the values of next or new step of time for all nodes were calculated, simultaneously.

The variation of the enthalpy $H$ allows to express the amount of heat involved during the transformation to constant pressure of a thermodynamic system [9]. Furthermore, the enthalpy depends on the temperature and this is a function of time.

$$
\frac{\partial H_{P C M}}{\partial t}=\frac{\partial H_{P C M}}{\partial T_{P C M}} \cdot \frac{\partial T_{P C M}}{\partial t}
$$

\section{Balance of heat energy in the PCM}

The variation of the thermal mass is equal to the transfer at the $\mathrm{PV} / \mathrm{PCM}$ interface, that is:

$$
V_{P C M} \frac{\partial H_{P C M}}{\partial t}=A h\left(T_{P V}-T_{P C M}\right)
$$

where $V$ is the volume. In this case, the enthalpy is a function of the fraction of molten PCM, is given as:

$$
\begin{gathered}
H_{P C M}=(1-f) \cdot \rho_{S} \cdot C p_{S} \cdot T_{M}+ \\
f \cdot \rho_{L} \cdot C p_{L} \cdot T_{M}+f \cdot \rho_{L} \cdot \Delta h_{M}
\end{gathered}
$$

when $f$ is molten fraction of material and may be expressed as:

$$
f=\frac{s(x)}{V_{P C M}}
$$

The solid-to-liquid phase transformation takes place at the melting temperature, if the temperature of the PCM is greater that its melting temperature, the PCM is completely liquid, such that $f$ is given as:

$$
f= \begin{cases}f=0 & T_{P C M}<T_{M} \\ f=1 & T_{P C M}<T_{M}\end{cases}
$$

Due to the fact that it is not possible to express the enthalpy $\mathrm{H}$ with respect to the temperature $\mathrm{T}$ as a linear function during the phase of latent heat, the equivalent heat capacity $C_{\text {eff }}$ in which the melting temperature $T_{M}$ is in the interface between the solid and liquid fraction was used [10]. The relation between enthalpy and temperature is shown in Fig. 4. when

$$
C_{e f f}= \begin{cases}C_{e f f}=\rho_{S} C p_{S} ; & T_{P C M}<T_{\min } \\ C_{e f f}=\rho_{L} C p_{L} ; & T_{P C M}>T_{\max } \\ C_{e f f}=\frac{\Delta h_{M}}{T_{\max }-T_{\min }} ; & T_{P C M}<T_{M} \text { and } T_{P C M}>T_{M}\end{cases}
$$

In summary, the energy balance of PCM is as follows:

$$
V_{P C M} C_{e f f} \frac{\partial T_{P C M}}{\partial t}=A \frac{\lambda_{L}}{s(x)}\left(T_{P V}-T_{P C M}\right)
$$

\section{Energy balance in the PV}

The variation of the thermal mass is equal to the sum of the loss in the air/PV interface, the transfer at the PV/PCM interface and the source of energy, that is: 


$$
\begin{gathered}
V_{P V} \rho_{P V} C p_{P V} \frac{\partial T_{P V}}{\partial t}=A h\left(T_{a m b}-T_{P V}\right)+ \\
A \frac{\lambda_{L}}{s(x)}\left(T_{P C M}-T_{P V}\right)+(1-\eta) A I
\end{gathered}
$$

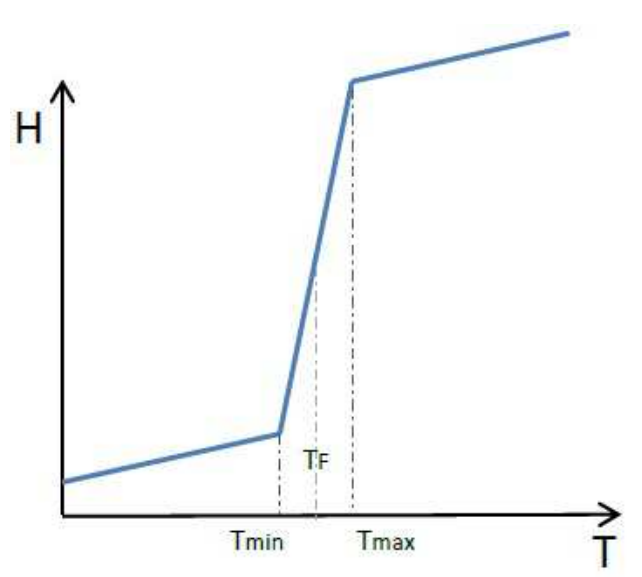

Figure 4. Enthalpy-temperature curve. The relation between enthalpy and temperature by phase change.

\section{Results}

The thermal evaluation of the PV/PCM system during the solar insolation was conducted. The temperature of the panel and the material was calculated using the propose models. The numerical simulation of the models was carried out in the Matlab software.

In the analyses of the energy balance, it can be noted that in the explicit and implicit models the variable $\mathrm{s}(\mathrm{x})$ is used, which is the thickness of the phase change material that is melted with regard to the time. In the models, the commercial paraffin Rubitherm RT42, with melting temperature of $42^{\circ} \mathrm{C}$, was used as PCM to filling the back of solar panel.

\subsection{Analytical Model}

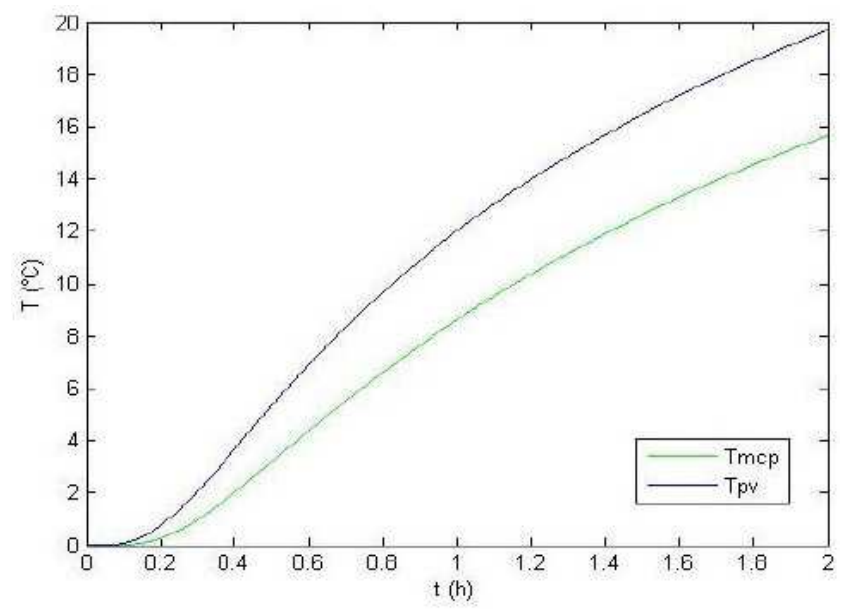

Figure 5. T-t curve of solid PCM and PV panel by analytical model.

The temperature of the PV/PCM interface was obtained of (6). The T-t curve is presented in Fig 5, in this is shown the difference of the temperature between the PCM in solid state and the PV, with regard to the time, during first hours of solar insolation. In this graph is observed that, when the temperature of $\mathrm{PCM}$ is of $14^{\circ} \mathrm{C}$, the temperature of $\mathrm{PV}$ is of $18^{\circ} \mathrm{C}$, that is, there is a temperature gradient of $4^{\circ} \mathrm{C}$ only for two hours of solar insolation, due to the fact that to the beginning of the day, the values of flow are small.

Based on the foregoing, if the hypothesis that the PCM and the $\mathrm{PV}$ are at the same temperature is done, the error is of $4^{\circ} \mathrm{C}$. The following models were considered this hypothesis.

\subsection{Explicit Model}

In the Fig. 6, the behavior of the temperature of the PCM as well as the temperature of the $\mathrm{PV}$, which reached $111^{\circ} \mathrm{C}$ after 12 hours of solar insolation, is observed.

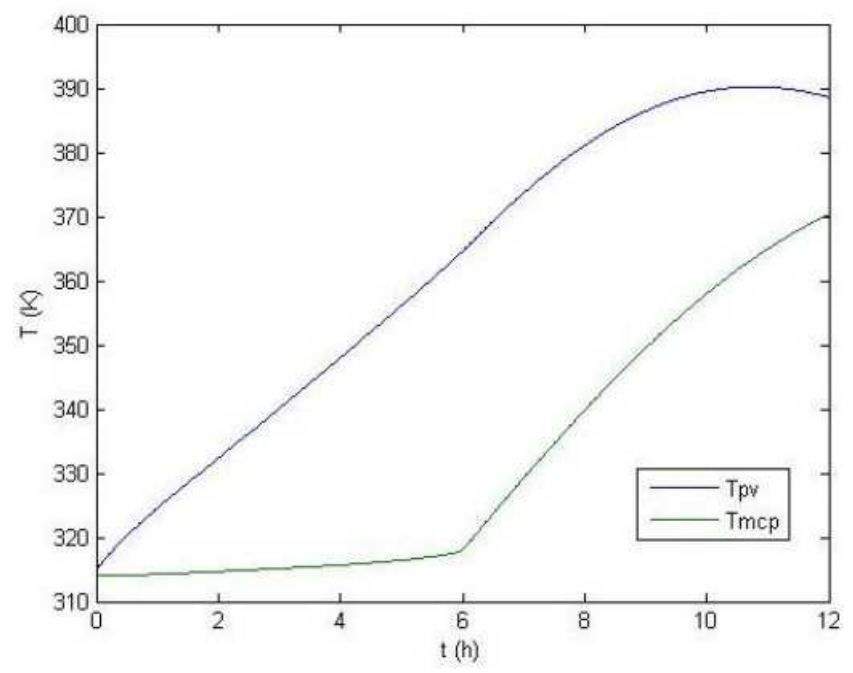

Figure 6. T-t curve of PCM and PV by the explicit model.

\subsection{Implicit Model}

To solve this problem, the implicit Euler method was used. At each time-step, an iterative procedure was performed and the convergence was assured by a criterion on the enthalpy of the PCM.

For analyze the behavior of the temperature of the PV, the simulation was carried out with two different PCMs. In principle, for the numerical simulation, the model was tested using a PCM with a melting temperature of $42{ }^{\circ} \mathrm{C}$. Furthermore, the commercial paraffin with a melting temperature of $27^{\circ} \mathrm{C}$ was used, because it is already known that for best performance, the maximum temperature of the photovoltaic panel must be $25^{\circ} \mathrm{C}$, then this is a condition for the use of the material RT27 as a different PCM in the analysis of the behavior of panel.

In the Fig. 7 and Fig 8, the behavior of the temperature of the PCM (RT42 and RT27 respectively) and the temperature of the PV is shown. In both figures, the temperature behavior of the material can be observed. In the Fig. 7 is observed that the temperature of the solar panel reached $107^{\circ} \mathrm{C}$ after 12 hours of exposure; this is $5^{\circ} \mathrm{C}$ more than the with material RT27 (see Fig 8). 


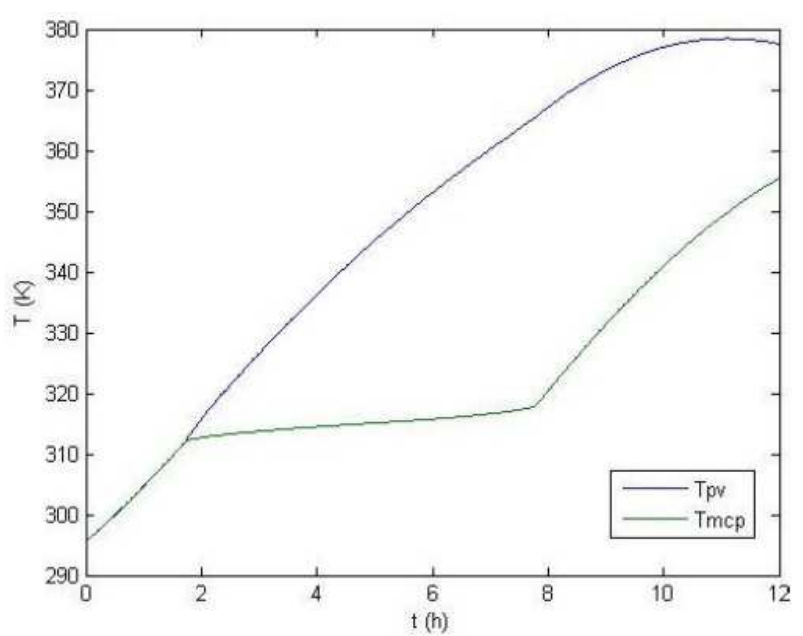

Figure 7. T-t curve of PCM RT42 ${ }^{\circ} \mathrm{C}$ and PV by the implicit model.

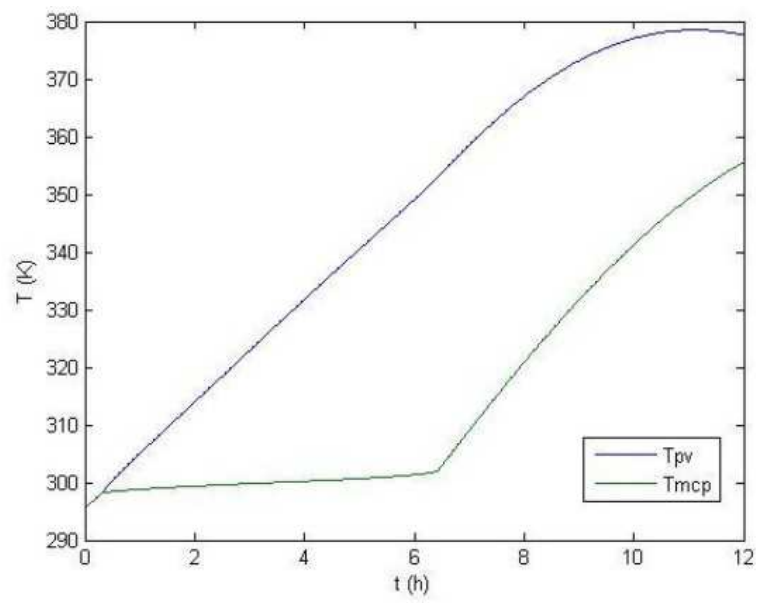

Figure 8. T-t curve of PCM RT2 ${ }^{\circ} \mathrm{C}$ and PV by the implicit model.

The results of the explicit and implicit mathematical models can be compared since they express the same phenomenon and use the same PCM. The Fig. 6, 7 and 8 show that the initial temperature of the panel is equal to the ambient temperature and with the time, it increases to the temperature of melting of the PCM. Depending on the quantity of material, the temperature remains almost constant during a time due to its ability to store energy, that is, to the latent heat. Later, when the PCM is completely liquid, its temperature continues to increase and in the same way the temperature of the PV, this is due to the phenomenon of conduction that exists between the liquid fraction of the material and the panel.

\section{Conclusions}

A thermal analysis of the cooling of a PV panel with PCM was carried out. In this study, three different models: analytical, explicit and implicit, were used to understand the behavior of the temperature of the system during the solar insolation. The following conclusions can be drawn:

- The temperature behavior, of both the PCM and the PV panel in the explicit and implicit models, follows the same trend. The temperature of the PV continued increasing even during the melting of the PCM.

- The conduction that exists between the material and the panel prevents the real cooling, that is, it exists a low efficiency of the panel.

- The size of the surface of exchange should be greater to increase the heat transfer and to avoid the increase of temperature of the panel.

- The complete melting of the material must be avoided. The PCM in solid state must always be in contact with the panel to absorb the heat generated and to keep the panel to a low temperature.

- The results obtained from the numerical analysis presented in this paper, should be considered as a first approach to the study of a PV / PCM system due to numerous simplifications introduced in the heat balances, as well as in the numerical method defined.

\section{Acknowledgements}

G. Santiago-Rosas wishes to express their gratitude to the Mexican National Council of Science and Technology (CONACYT) for its financial support throughout of Master of Science Program.

M. Flores-Domínguez would like to thank for the financial support received from Council of Science and Technology of Mexico State (COMECYT), Mexico during the realization of this work.

\section{Nomenclature}

$\begin{array}{ll}C_{e f f} & \text { Equivalent thermal capacity } \\ C p & \text { Specific heat capacity } \\ f & \text { Molten fraction } \\ h & \text { Coefficient of natural convection } \\ H & \text { Enthalpy } \\ I & \text { Solar insolation } \\ M & \text { Mass } \\ s(x) & \text { Thickness of material } \\ S & \text { Surface } \\ t & \text { Time } \\ T & \text { Temperature } \\ V & \text { Volume } \\ \Delta h & \text { Latent heat } \\ \text { Greek letters } & \\ \rho & \text { Density } \\ \lambda & \text { Heat conductivity } \\ \eta & \text { Performance of PV } \\ \text { Subscripts } & \\ \text { L } & \text { Liquid phase } \\ \mathrm{S} & \text { Solid phase } \\ \mathrm{M} & \text { Molten phase } \\ & \end{array}$

\section{References}

[1] H. G. Teo, H., P. S. Lee, M. N. A Hawlader, "An active cooling system for photovoltaic modules," Applied Energy, vol. 90, no 1, pp. 309-315, 2012. 
[2] W. He, Y. Zhang, J. Li, "Comparative experiment study on photovoltaic and thermal solar system under natural circulation of water," Applied Thermal Engineering, 31(16), pp. 3369-3376, Nov. 2011.

[3] M. J. Huang, P. C. Earnes, B. Norton, "Natural convection in an internally finned phase change material heat sink for the thermal management of photovoltaics," Solar Energy and Solar Cells, vol. 95, no 7, pp. 1598-1603, 2011.

[4] B. Vidal Jiménez, "Modelización del cambio de fase sólido-líquido. Aplicación a sistemas de acumulación de energía térmica," s.1.: Centro Tecnológico de Transferencia de Calor, Depto.de Máquinas y motores térmicos, Universidad Politécnica de Cataluña, 2007.

[5] M. J. Huang, P. C. Earnes, B. Norton, "Thermal regulations of building-integrated photovoltaics using phase change materials," International Journal of Heat and Mass Transfer, vol. 47, no 12, pp. 2415-2733, 2004.
[6] J. P. Braun, B. Faraggi, A. Labouoret, "Les cellules solaires", ETSF (Editions Techniques et Scientifiques Françaises) - Paris, 1996.

[7] J. P. Nadeau, "Energetique Industrielle". Talence: Art et Métiers-ParisTech, 2009.

[8] Y. Jannot, “Transformation de Laplace inverse", En: Transferts thermiques, p. 113, 2002.

[9] H. Mehling, L. F. Cabeza, Heat and cold storage with PCM Handbook, Publisher Springer, Germany, 2008.

[10] F. Rouault, "Analyse expérimentale et modélisation d'un échangeur-stockeur contenant des matériaux à changement de phase," Congrès SFT (Société Française de Thermique), Talence, France, 2012. 